\title{
A New Lane Departure Warning Algorithm Considering the Driver's Behavior Characteristics
}

\author{
Lun Hui Xu, ${ }^{1}$ San Gen Hu, ${ }^{1}$ and Qiang Luo ${ }^{2}$ \\ ${ }^{1}$ School of Civil Engineering and Transportation, South China University of Technology, Guangzhou, Guangdong 510640, China \\ ${ }^{2}$ School of Civil Engineering, Guangzhou University, Guangdong 510006, China
}

Correspondence should be addressed to San Gen Hu; husangen2010@163.com

Received 6 May 2015; Revised 7 July 2015; Accepted 26 July 2015

Academic Editor: Gregory Chagnon

Copyright (C) 2015 Lun Hui Xu et al. This is an open access article distributed under the Creative Commons Attribution License, which permits unrestricted use, distribution, and reproduction in any medium, provided the original work is properly cited.

In order to meet the driving safety warning required for different driver types and situations, a new lane departure warning (LDW) algorithm was proposed. Its adaptability is much better through setting the different thresholds of time to lane crossing (TLC) using fuzzy control method for driver with different driving behaviors in different lanes and different vehicle movements. To ensure the accuracy of computation of TLC under the different actual driving scenarios, the algorithm was established based on vehicle kinematics and advanced mathematics compared to other ways of computation of TLC. On this basis, a LDW strategy determining driver's intentions was presented by introducing identifying vehicle movements. Finally, a vast quantity of the real vehicle experiments was given to demonstrate the effectiveness of the proposed LDW algorithm. The results of the tests show that the algorithm can decrease false alarm rate effectively because of distinguishing from unconscious by real-time vehicle movements, and promote the adaptability to the driver behavior characteristics, so it has favorable driver acceptance and strong intelligence.

\section{Introduction}

Traffic accidents have become the first public nuisance of modern society all over the world, which caused great loss of the national people's lives and property. In our country, although the amount of road traffic accidents and deaths has declined after relevant management departments took joint efforts in recent years, the road safety situation was still serious. According to the latest statistics from the National Bureau of Statistics of China, there were at least 200,000 road traffic accidents, 70,000 deaths, and 70,000 direct economic losses every year. In this paper, the statistical data of road traffic accidents and deaths as well as direct economic losses was presented between 2004 and 2013, as shown in Figure 1.

According to the research results of related literature at home and abroad, the $80-90 \%$ of road traffic accidents were caused by the driver behavior [1]. In a word, drivers' inattention resulting from various causes was an important reason for frequent traffic accidents. Drowsiness, veering off the road, and inattention account for a large proportion in traffic accidents, according to the analysis of the causes of traffic accidents causation. For the most causes of driver factors, they are avoidable. The road traffic accidents caused by the real-end collision hold about $30 \%$ of total accidents. And, more remarkably, single vehicle roadway departure (SVRD) crashes account for approximately $20 \%$ of all types of vehicle crashes in the US $[2,3]$. Therefore, how to avoid the traffic accidents caused by lane departure has been paid more and more attention, since they spend a little share of total traffic accidents that cannot be ignored. According to the Federal Highway Administration Research, lane departure warning system (LDWS) can avoid the $30-70 \%$ traffic accidents resulting from lane departure. The LDWS was intended to alert drivers to departure situations caused by drowsiness, alcohol, or distraction and so on, anticipating that drivers will make immediate adjustments in their direction, speed, or both to avoid accidents, which was considered an integral part of driver assistant system. All in all, the study of LDWS played an important role in improving the road traffic safety and reducing the number of deaths and economic loss of traffic accidents.

According to our review, a method known as time to lane crossing (TLC) is feasible and intelligent as compared 


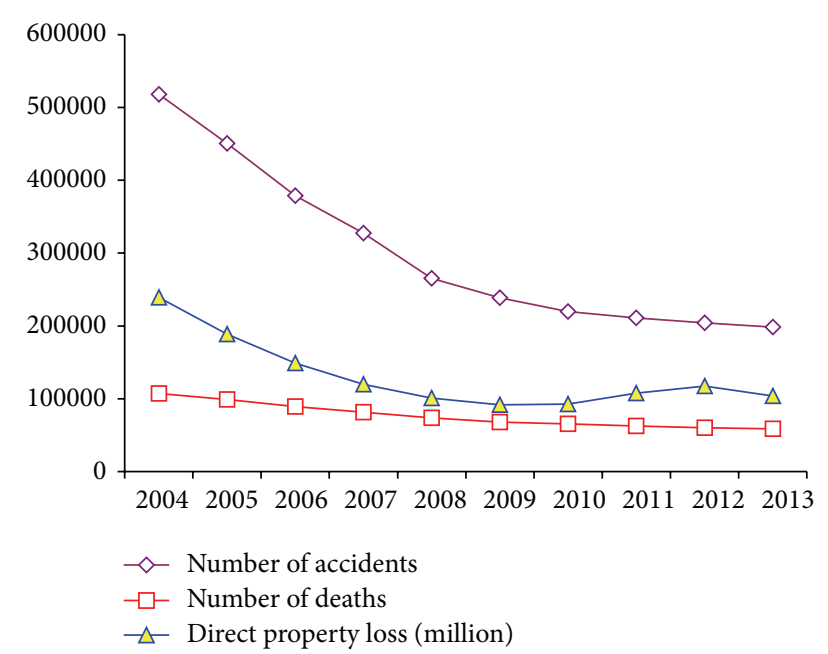

FIGURE 1: Statistical data of road traffic accidents, deaths, and direct economic losses among 2004 and 2013.

with RRS-based [4], VRBS-based [5], CCP-based, and FODbased [6] approach in terms of reducing false warnings and increasing warning anticipation. The study of Kreucher et al. [7] computed the TLC by a time dependent function of the lateral distance to lane boundary. Batavia et al. [8] used a memory based on method to calculate the TLC. A prior trained array of frequency distribution of future lateral vehicle positions related to current position and velocity served as a basis to the TLC calculation. Batavia proposed an extension to the TLC and RRS idea of allowing the driver to drift beyond the lane border by adding a virtual lane boundary with adjusting by a learning phase. However, a major problem with the existing TLC-based approach is the complexity of TLC computation in real time, the fixed or static threshold, and the straight of warning algorithm; because of that, LDWS based on TLC algorithm could not meet different types of driver and situation for the driving safety warning required. Obviously, because of the differences in driver individuals, the reaction time and mental sharpness are different on different lanes or under different speed. Therefore, the existing LDWS using the fixed TLC threshold for different drivers is unreasonable due to neglecting driving styles. One caveat is that a fatal disadvantage with it is of no use for some drivers, which even brings inconvenience to the normal driving process. The LDWS not only cannot play the role of security early warning, but also may lead to new potential danger. In addition, the LDWS may also bring a high false alarm by reason of the simple alarm logic which may not identify vehicle movements and consider driver's intention, for example, lane changing or turn.

From the above, it is essential to develop a new lane departure warning algorithm for driver security needs while driving on the road. In order to reduce false alarm rate, how to compute the TLC, set the time threshold, and lay down the warning strategies is the core as well as the main contribution of the paper. They are explained in more detail one by one in the next section. First up, the computation of TLC is described.

\section{Computation of TLC}

2.1. Historical Review. The TLC (time to lane crossing) means the time until one of the vehicle tires crosses the lane boundaries [9]. An important application of TLC in lane departure warning system is to detect instances when the vehicle actually moves out of the lane and to warn the driver in order to avoid an immediate accident and incident [10]. There are different ways to approximate TLC during vehicle driving [11].

Algorithm 1. The first method to approximate TLC was based on the assumption that the vehicle's lateral velocity was a constant over a short period of time. The method took the vehicle's current lateral position and lateral velocity, which was expressed as follows:

$$
T_{u}=\frac{D}{v_{\mathrm{la}}}
$$

with $D$ as the distance between the outside edge of the tire and lane boundary and $v_{\mathrm{la}}$ as the vehicle lateral velocity. $D$ was calculated as

$$
D=\frac{w_{l}-w_{v}}{2}-d
$$

of which $w_{l}$ was the width of lane; $w_{v}$ was the width of vehicle; $d$ was lateral position of the centerline of the vehicle relative to the center of the lane.

The big advantage of this method was that it utilized only easy to measure variables and warned earlier if the vehicle was departing from the road more quickly. But it was not quite correct at all times, since it assumed the vehicle's lateral velocity would be constant over a short period, which is not true, depending on drivers' driving state and the upcoming road geometry. If the road curvature was changing and/or the driver was turning or negotiating a curve, the lateral velocity over time would vary.

Algorithm 2. The second way to approximate TLC was that it took vehicle lateral acceleration into account over the 1storder method, which assumed that vehicle lateral acceleration would keep constant over a short time. The formula was shown as the following:

$$
\begin{aligned}
T_{a}=\frac{\left(-v_{\mathrm{la}}+\sqrt{v_{\mathrm{la}}^{2}+2 a_{\mathrm{la}} D}\right)}{a_{\mathrm{la}}} \\
\left(a_{\mathrm{la}} \neq 0, \text { otherwise, use 1st order method }\right),
\end{aligned}
$$

with $a_{\mathrm{la}}$ being the vehicle lateral acceleration towards the edge of the lane.

The major advantage of this way over the 1st-order method was that it warned earlier and computed more accurately because of considering the vehicle trajectory relative to the lane and lateral acceleration information, instead of simply being determined by the current lateral velocity. Be warned that the vehicle lateral acceleration was constant over 
time, which may not be sure, only if the driver kept a constant steering, such as fixed yaw rate.

Algorithm 3. The third algorithm to compute TLC was what considered more additional information about the road geometry and vehicle trajectory, such as vehicle forward velocity, yaw angle relative to the lane centerline, the radius of curvature, and the radius of road, which was expressed as follows:

$$
T_{d}=\frac{-v \tan \alpha+\sqrt{(v \tan \alpha)^{2}+2 v^{2} D\left(1 / R_{r}-1 / R_{v}\right)}}{v^{2}\left(1 / R_{r}-1 / R_{v}\right)},
$$

with $v$ being vehicle forward velocity; $\alpha$ being yaw angle of vehicle relative to the lane centerline; $R_{r}$ being radius of road; and $R_{v}$ being radius of trajectory.

This algorithm would provide a more accurate approximate of the TLC than the previous algorithms and also generate an earlier warning on the approach to a curve, since it considered the road geometry and vehicle trajectory. However, this algorithm required additional sensor so as to get more parameters that the previous algorithms did not require. And the major problem is as follows: this algorithm may be more sensitive to errors in sensor estimates than the algorithm mentioned above.

Algorithm 4. The fourth computation of TLC took the vehicle's distance traveled towards the edge of the lane and forward velocity of vehicle, as shown in the following [12]:

$$
T_{1}=\frac{\mathrm{DLC}}{v},
$$

with DLC as the distance to line crossing along the vehicle path and $v$ as the vehicle speed.

This algorithm was presented from the longitudinal perspective, whereas the above were depicted from the lateral perspective. In general, the DLC was solved trigonometrically using the cosine rule, and the vehicle's forward velocity can be seen as a constant over a short period of time during unintentional drifting towards the lane boundary.

2.2. Algorithm Development. This section develops a new algorithm to calculate the TLC based on vehicle kinematics and advanced mathematics. This algorithm mainly builds mathematical model of vehicle movement in nature. It does not require a lot of assumptions and more additional parameters except the vehicle's forward velocity and yaw angle relative to the lane centerline which can be measured accurately easier. As will be seen in the following experiment, this method does a good job at calculating TLC, whether on straight roads or roads with constant curvature.

In short, this algorithm can be presented where the lateral distance vehicle driving until one of the tires crosses the lane boundary over a TLC is $D$. Owing to the lateral velocity timevarying instead of constant, TLC is calculated by the integral calculation. Note that the only assumption this algorithm makes is that the travel speed is a constant over a period of

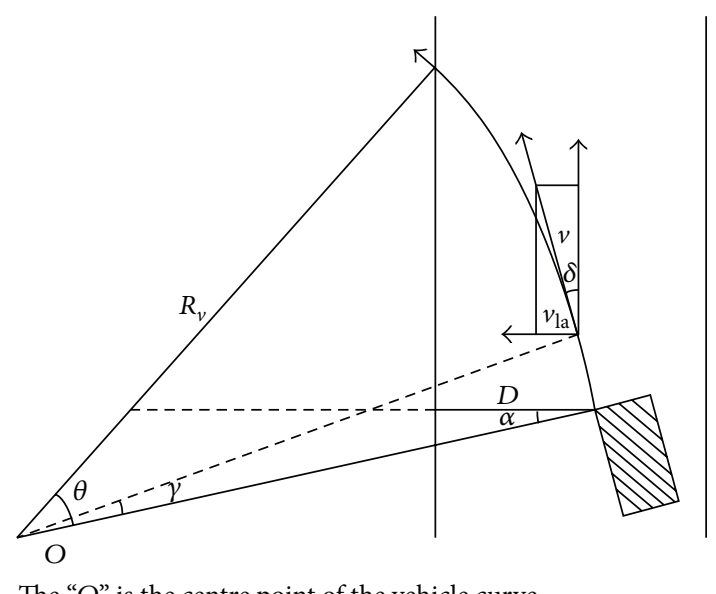

The "O" is the centre point of the vehicle curve.

FIgURE 2: Computation of TLC for negotiating a curve on straight road.

time (over the next one second or so), which is accordant with the practical situation. First, the case for straight roads is described; see Figure 2. In general,

$$
\int_{0}^{T_{\mathrm{lc}}} v_{\mathrm{la}} d t=D
$$

where $T_{\mathrm{lc}}$ is TLC. The time-varying lateral velocity, $v_{\mathrm{la}}$, is calculated as

$$
v_{\mathrm{la}}=v \sin \delta, \quad \delta=\gamma+\alpha,
$$

where $\gamma$ is the degree as vehicle crosses at time $t$; if $t=T_{\mathrm{lc}}$, $\gamma=\theta$; and $\alpha$ is yaw angle of vehicle to the lane centerline

$$
\theta=w t=\frac{v}{R_{v}} t
$$

of which $R_{v}$ is radius of the vehicle path.

By the comprehensive analysis above, $T_{\mathrm{lc}}$ is computed as

$$
T_{\mathrm{lc}}=\frac{R_{v}\left(\arccos \left(\cos \alpha-D / R_{v}\right)-\alpha\right)}{v} .
$$

Figure 3 shows how to calculate TLC while the vehicle is negotiating a curve on curved road. Again, TLC is calculated as (6). However, in this case $\delta$ is calculated differently. It can be computed with cosine rule according to

$$
\delta=\arccos \left(\frac{\left(R^{2}+R_{v}^{2}-D_{r-v}^{2}\right)}{\left(2 R R_{v}\right)}\right)
$$

with $D_{r-v}$ being the distance between the current point of the road curve and the centre point of the vehicle curve which can be computed by (11) and $R$ being the distance from the vehicle tire to the current point of the road curve which can be computed by (12):

$$
\cos \alpha=\frac{\left(R_{v}^{2}+\left(R_{r}+D\right)^{2}-D_{r-v}^{2}\right)}{\left(2 R_{v}\left(R_{r}+D\right)\right)}
$$




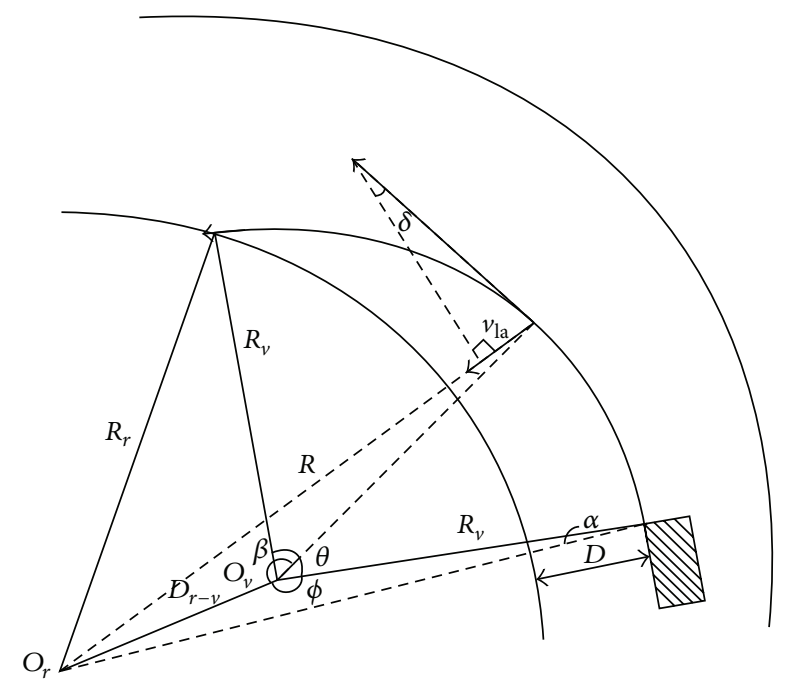

The point $O_{v}$ is the centre point of the vehicle curve; the point $O_{r}$ is the centre point of the road curve.

FIgURE 3: Computation of TLC for negotiating a curve on curved road.

with $\alpha$ being yaw angle of vehicle to the lane centerline:

$$
\cos \beta=\frac{\left(D_{r-v}^{2}+R_{v}^{2}-R^{2}\right)}{\left(2 D_{r-v} R_{v}\right)},
$$

with $\beta=2 \pi-\phi-\left(v / R_{v}\right) t$, and that $\phi$ is computed as

$$
\phi=\arccos \left(\frac{\left(D_{r-v}^{2}+R_{v}^{2}-\left(R_{r}+D\right)^{2}\right)}{\left(2 \cdot D_{r-v} \cdot R_{v}\right)}\right) .
$$

2.3. Algorithm Validation and Discussion. In this section, the proposed algorithm of TLC computation is tested and compared with the abovementioned (Algorithms 1 3). They are compared on a set of typical driving scenarios, depending on how accurately they approximate the TLC.

The baseline parameters used in the following analysis include

(i) lane width which is $3.6 \mathrm{~m}$,

(ii) vehicle width which is $1.8 \mathrm{~m}$,

(iii) vehicle velocity which is $25 \mathrm{~m} / \mathrm{sec}$,

(iv) vehicle which starts out centered in the lane and traveling to the lane (except in Scenarios 2, 5, 6, and 9),

whereas the lateral position of the centerline of the vehicle to the center of the lane is zero.

The ten scenarios modeled in this analysis are shown below. They are meant to cover a fairly representative range or road geometry/vehicle trajectory situation encountered in the real word. The result of TLC computation is in the right pane of every scenario in Figure 4. In this part, TLC computation of the proposed algorithm in this paper is referred to as $T_{\mathrm{lc}}$.
Similarly, Algorithm 1 is referred to as $T_{u}$, Algorithm 2 is referred to as $T_{a}$, and Algorithm 3 is referred to as $T_{d}$.

The actual TLC and calculated value based on the previous algorithms were recorded by the real vehicle experiment. Owing to consider drive safety, the experiment was established with lane changing in different direction to simulate lane edge crossing. In this experiment, the forward computervision-based lane trackers were used to detect the lane markings and estimate vehicle's position in real time. This approach includes a robust real-time lane-detection-andtracking algorithm for local roads and freeways of various challenging scenarios, such as a lane curvature, worn lane markings, and lane changes [13]. In addition, the parameters required of TLC algorithm could be obtained by some sensors. Travel speed of the vehicle was relatively easy to sense by the speedometer. The yaw rate was relatively straightforward to measure by a yaw rate gyro with a sufficiently fast update rate and accuracy. The vehicle yaw angle was somewhat difficult to measure with a forward computer-vision since this approach required distinguishing between vehicle yaw angle and road curvature, both of which had very similar effects on the appearance of the road ahead in a forward camera image. For the same reason, road radius of curvature was also hard to measure. In order to solve this problem, the differential GPS and GIS were used. The former would provide the vehicle's current heading and position. The latter could provide the upcoming road curvature according to the vehicle's current heading and position. Obviously, the difference between the heading of the vehicle and the heading of the road is the vehicle's yaw angle. This equipment, sensors, and commercial software taken by Traffic Engineering Lab were used in this paper.

It is found that the proposed algorithm and Algorithm 3 estimate TLC more accurately than Algorithm 1 as well as Algorithm 2 in the scenarios shown in Figure 4 except Scenarios 1 and 2, as shown in Figure 4. In each of the other eight scenarios, Algorithm 1 overestimates the true TLC. Sometimes the maximum error is over 4.165. The reason for that is Algorithm 1 does not take the road curve and/or vehicle trajectory curve into account, which makes the lateral velocity change over time instead of being constant. Algorithm 2 computes TLC almost perfectly in the scenarios but Scenarios 6, 8, and 9, by relaxing the assumption that lateral velocity must be constant over time replaced by a constant lateral acceleration. However, the error increases somewhat on Scenarios 6, 8, and 9. Algorithm 3 is slightly more precise than Algorithms 1 and 2, as the proposed algorithm does. And yet for all that, Algorithms 1, 2, and 3 are not perfect method at a practical angle. There are reasons: lateral velocity and lateral acceleration for Algorithms 1 and 2 are hard to calculate accurately, which can be obtained indirectly as the first and second derivative of lateral distance, and the error of them gets compounded once and twice. The more sophisticated Algorithm 3 is, the more sensitive to sensor error it will be. One caveat is that some of its parameters cannot be sufficiently measured by sensors on some vehicles today. However, the established algorithm in this paper is not the case. 


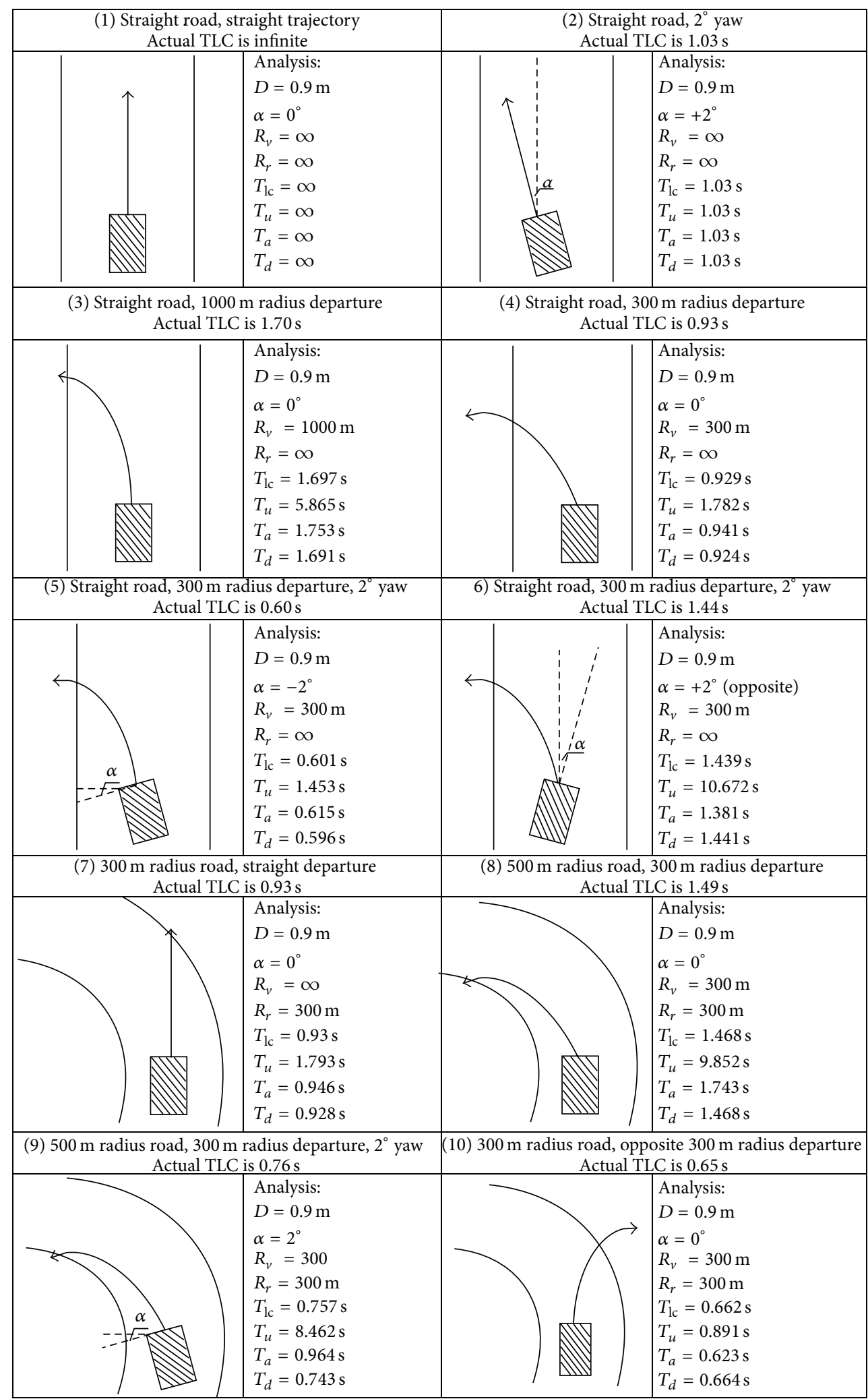

FIGURE 4: Computation of TLC on different driving scenarios. 


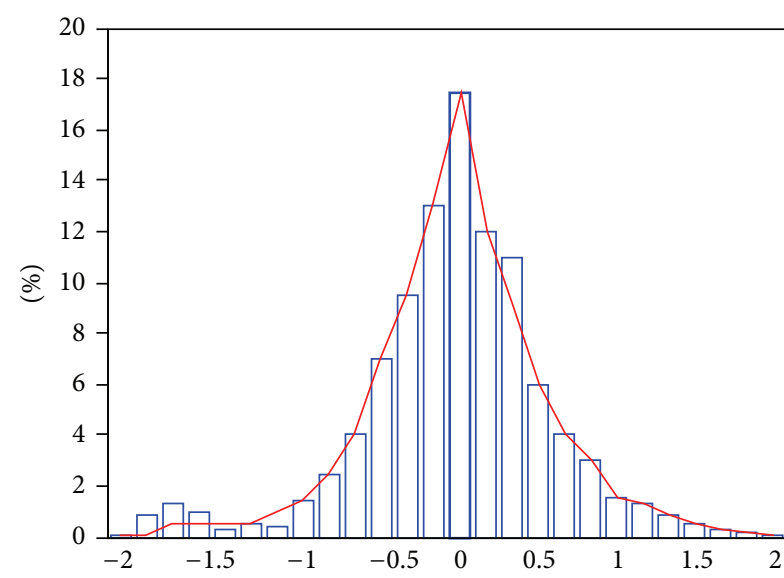

(a) Lane 1

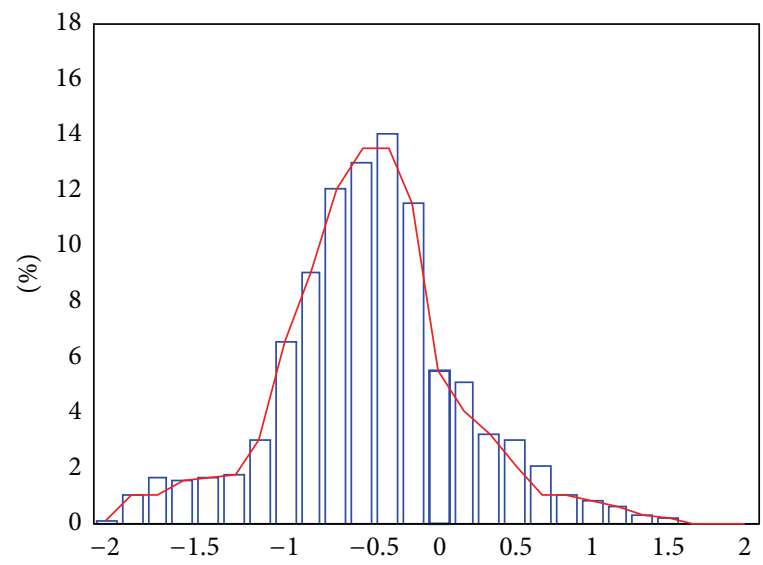

(c) Lane 3

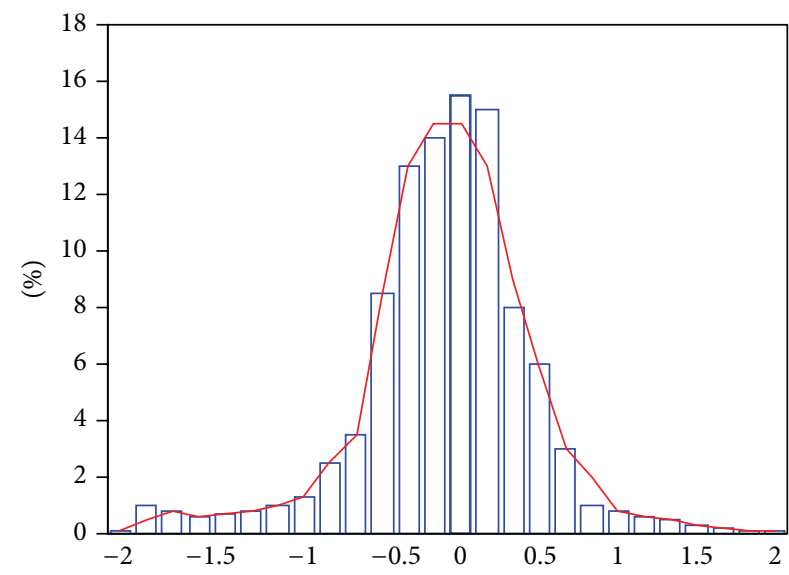

(b) Lane 2

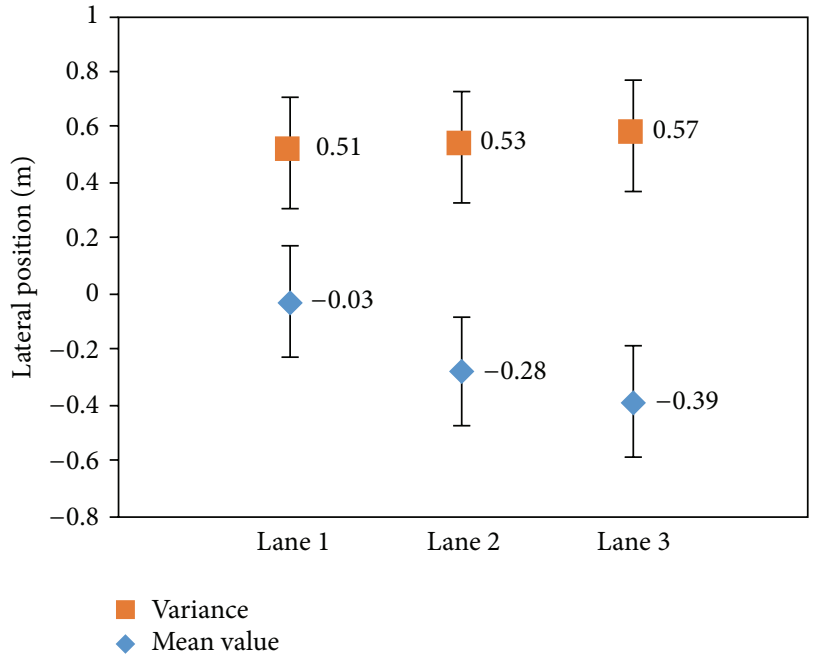

(d) Different lane

FIGURE 5: Statistics histogram of lateral position on the Guangzhou freeway and mean and variance of lateral position.

\section{Time Threshold Setting}

It is clear that each driver has his own driving habits. For example, some of the drivers are used to driving on the left lane and some are not. In that case, setting the same threshold of time to lane crossing (TLC) is particularly unreasonable. Even if the driver moves off the same lane from two different directions (left, right), the time threshold may be not the same because of different travel time to the left lane boundary and to the right lane boundary. In general, the time threshold sets in terms of the vehicle lateral position on lane. Therefore, a large quantity of real vehicle experiments is presented in which the different lateral position features on different lanes form different directions for different drivers are assessed in order to offer the guidance for time threshold setting. In the first experiment lateral position feature of different lanes is obtained. The second experiment finds that lateral position feature is not the same for all participants. These are explained in more detail in the next section.
3.1. Lateral Position Feature on Different Lanes. The experiment was performed on the real Guangzhou freeway, where its traffic was light in which the density of traffic was low to allow vehicles to travel at the desired speed allowed. All roads consisting of three lanes were not bad and alternated between straight road and curved road. Lane width was $3.6 \mathrm{~m}$.

Twenty experienced drivers participated in the experiment. All were between 25 and 50 years of age, sixteen males and four females. They were asked to drive at the desired speed allowed on the basis of their driving habits and could change lane, turn, and negotiate a curve according to actual road traffic condition. All data of sampling periods was 0.1 seconds. The experiment remained at least 40 minutes.

Figures 5(a) 5(c) represented the distribution of lateral position on three different lanes. Figure 5(d) depicted the mean and variance of the lateral position on three different lanes.

Figures $5(\mathrm{a}) \sim 5(\mathrm{~d})$ showed the mean of lateral position was negative and the variance of lateral position was different 


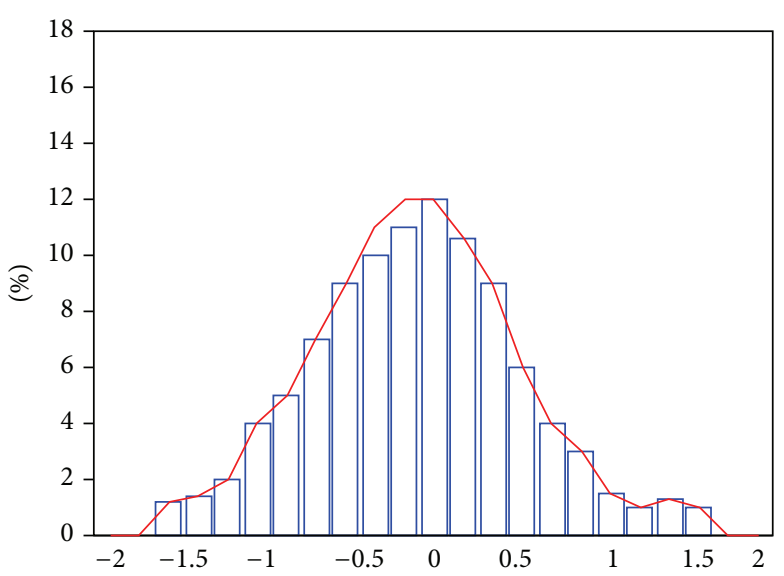

(a) Adventure type

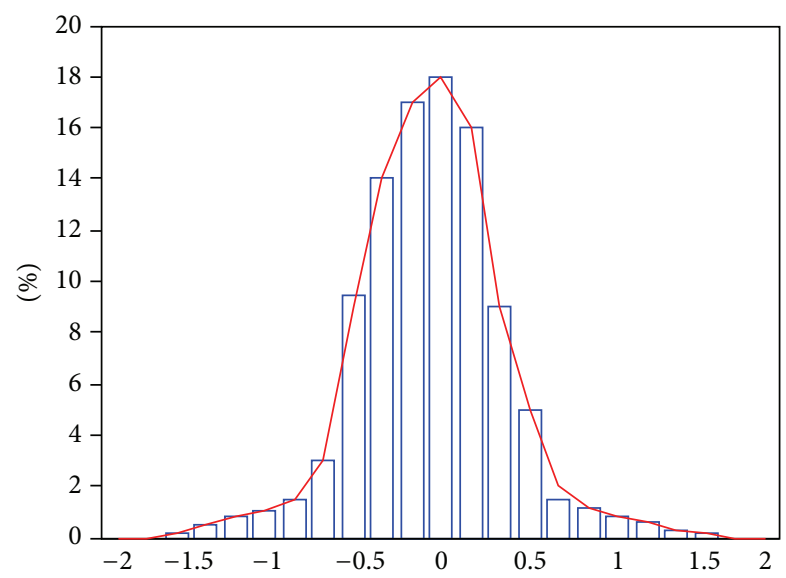

(c) Tight type

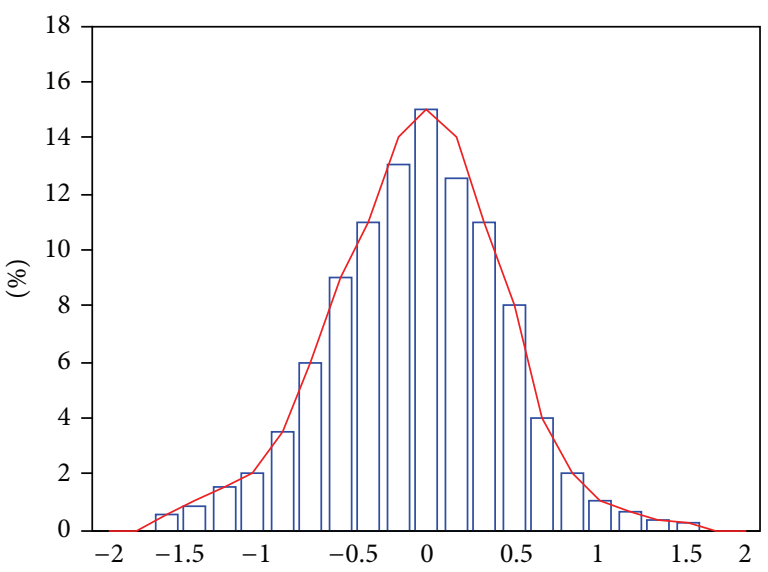

(b) Normal type

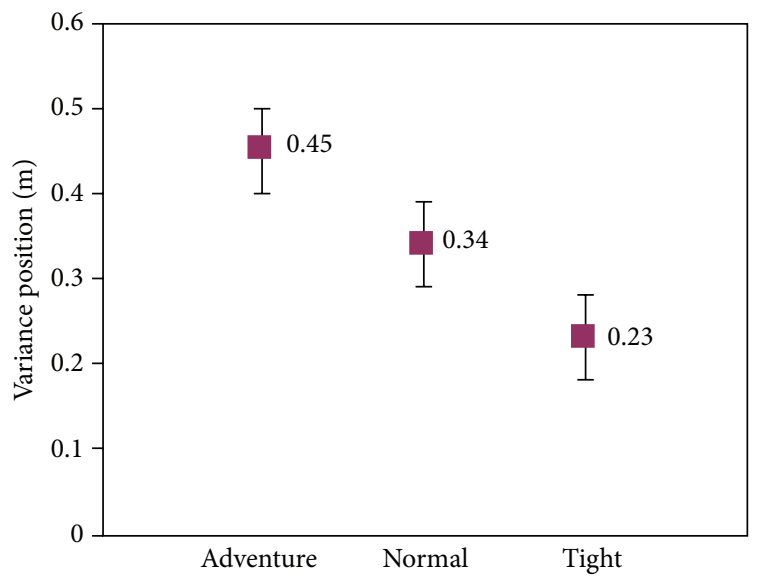

(d) Different driver

FIGURE 6: Statistics histogram of lateral position for three different types of drivers.

on three different lanes, which increased gradually with lane change from the left (Lane 1) to the right lane (Lane 3). It was concluded that the drivers were used to driving on the left side of lane. And if the current lane was farther away from the left lane, this phenomenon was more obvious. So, the time to different lane crossing or to left/right boundary of lane was not the same under the same road traffic condition, which implies the time threshold required was different in order to accommodate that situation. Set some rules as follows:

(1) The time threshold is higher for departure from the left side of lane than opposite direction on the same lane. The main reason is that almost all of drivers are used to driving on the left side of lane in our nation, where the amplitude of the steering wheel to the right is larger than that to the opposite direction.

(2) The time threshold is different on the different lanes. From Lane 1 to Lane 3 , the time threshold for departure from the left side of lane is on the decline, whereas it is on the rise for opposite direction.

3.2. Lateral Position Feature on Different Drivers. The same equipment was used as in experiment 1 , as well as the same place and experienced drivers. From the view of social psychology, the mechanism of driving behavior was analyzed and studied by an investigation among drivers. And the drivers could be classified into three groups: tight, normal, and adventure. The numbers were 7,8 , and 5 , respectively. They were also asked to drive at a speed as they would on the lane and not change lane. The experiment remained at least 40 minutes.

Figure 6 presented the distribution of lateral position of the centerline of the vehicle relative to the center of the lane for three different types of drivers under normal lane keeping.

The distributions of the lateral position conform to the law of normal distribution in Figures 6(a) 6(c). For the "tight" driver, the normal distribution curve was the steepest, which indicated the vehicle driving along the lane center most of time, the next was the "normal" driver, and the "adventure" driver was the last. The variance of the lateral position could explain the amplitude of veering off the center of lane for three different driver types as data signatures (see Figure 6(d)). There are some reasons that could be given: the "tight" driver could try to keep driving along the lane center owing to his high focused attention, whereas the "adventure" driver mostly wandered on a straight road because of his looseness 
or bravery, while the normal driver usually behaved between these two occasions.

To sum up, in order to decrease false alarm rate, it is significant that the different thresholds of TLC are set when drivers with different driving behaviors are on different lanes and departure directions (direction right, direction left). It is very difficult to calculate the time thresholds by mathematical models because of the variety and nonlinearity in different situations. Therefore, it can be determined by the fuzzy inference method, where there is a big advantage on solving that case.

\subsection{Time Threshold Decision Based on Fuzzy Control Method.}

This section gives details of fuzzy-logic structure utilized for decision of time threshold setting. Rule generation and input and output membership function formation, along with the adaptation and intelligent TLC thresholds output, are covered. Through the above analysis, we can see that the TLC thresholds are mostly determined by some key parameters, which are driving style, lane style, and departure direction. Therefore, driving style $T_{d}$, lane style $L_{d}$, and departure direction $D_{d}$ are chosen as inputs of the decision system. The output variable is the TLC thresholds.

First, every input variable should be fuzzified in order to be treated by the fuzzy rule base. The input space $U$ is simply the expected working range to be included in the fuzzy set. For the driving style $T_{d}, U$ is defined as $U_{T_{d}}=[0.23,0.45] \mathrm{m}$. It can be represented by lateral position standard deviation, which can be calculated by utilizing long-term driving data from each driver. This is explained in more detail in Section 3.2. There are three input membership functions, which are represented by fuzzy sets "small" (tight), "medium" (normal), and "large" (adventure) for driving style variable. Likewise, for the lane style $L_{d}, U$ is defined as $U_{L_{d}}=[1,3]$. There are also three input membership functions, represented by fuzzy sets "small" (right lane), "medium" (middle lane), and "large" (left lane) for lane style variable. For the departure direction $D_{d}, U$ is defined as $U_{D_{d}}=[-0.9,0.9] \mathrm{m}$, which represents the distance between the outside edge of the tire and the center of the lane. Unlike previous analysis, the $D_{d}$ input has two membership functions representing the fuzzy sets "left" and "right." It is worth mentioning that the expected range of every input variable may exceed or be less than $U$. Values outside the expected ranges are set to the nearest range extremum. If $T_{d}<0.23 \mathrm{~m}$, let $T_{d}=0.23 \mathrm{~m}$. Likewise, if $T_{d}>0.45 \mathrm{~m}$, let $T_{d}=0.45 \mathrm{~m}$. The output of fuzzy rule base, TLC thresholds $T_{t}$, has three fuzzy membership functions representing fuzzy sets "small (S)," "medium (M)," and "large (L)." The TLC thresholds ranges are achieved to be $(0.7,2) \mathrm{s}$. If the threshold is smaller than $0.7 \mathrm{~s}$, it may be too short for the driver to respond in emergency situations. If the threshold is larger than $2 \mathrm{~s}$, it may generate superfluous alarming. The selection basis is described in more detail elsewhere $[14,15]$.

Second, the membership function of fuzzy inference method adopts the normal distribution function and triangle function in this paper, considering that membership functions need to demonstrate the impact of corresponding input and output variables as fully as possible and meanwhile combining with simulation analyses of different fuzzy
TABLE 1: Rules used to determine the TLC thresholds.

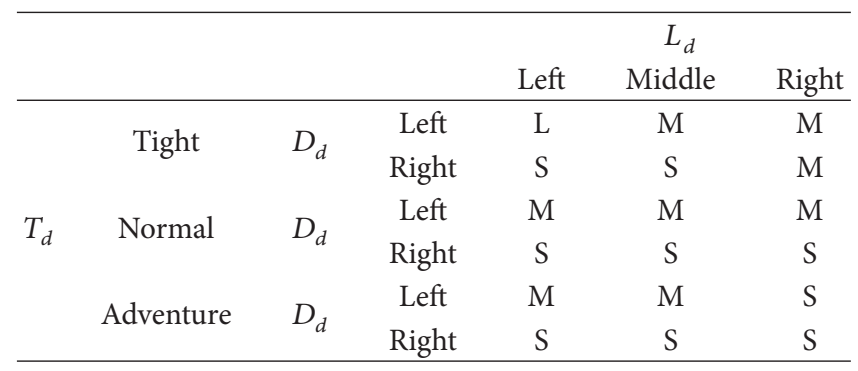

membership functions (e.g., sigmoid membership function, triangle membership function, and normal distribution membership function) [16]. In this case, this study uses the input membership functions for $T_{d}, L_{d}$, and $D_{d}$, which are shown in Figures $7(\mathrm{a})-7(\mathrm{c})$. The trapezoidal membership functions are used for output variables $T_{t}$, as shown in Figure 8.

Third, the supervisory approach using a rule-based system is used. The selection of a rule-based system is guided by the need to express heuristics and human expertise employed during driving experiment. The rules can be summarized in a rule set, as shown in Table 1 . The "tight" drivers have a large TLC threshold for left departure in left lane, indicating that the "tight" driver needs alarm earlier in order to make immediate adjustments in current direction, speed, or both. On the contrary, the "adventure" drivers have a small TLC threshold in the above situation. The "normal" drivers are between "tight" drivers and "adventure" drivers. The rules are interpreted using IF (antecedent) and THEN (consequent); for example, IF $T_{d}$ is tight, $D_{d}$ is left, and $L_{d}$ is left, THEN $T_{t}$ is large.

\section{Establishment of LDW Strategy}

In this section, a more detailed warning strategy of the proposed LDW algorithm in this paper will be described. The main idea is if time to the lane crossing falls below a threshold, a warning is triggered, which is well described by the following formula:

$$
\text { if } \mathrm{TLC}<T_{\mathrm{lc}} \Longrightarrow \text { warn. }
$$

In order to decrease false alarm rate, it is necessary to introduce the driving intention identifying to the warning strategy by vehicle movements, so that the conscious lane departure (such as wandering) can be distinguished from unintentional lane departure (such as changing lanes, negotiating a curve, or making turn). The following determines whether the vehicle is going straight/wandering or changing lanes, negotiating a curve, or making turn by some typically data signatures (such as heading angle, lateral distance, and curve's radius).

4.1. Lane Change Steering. Two variables were used to identify a lane change: a change in lateral position and the heading angle. The lateral position threshold was set based on the width of typical lane $(3.6 \mathrm{~m})$ and the width of vehicle (about $1.8 \mathrm{~m}$ ). Thus, a change in lateral position between $1.8 \mathrm{~m}$ 


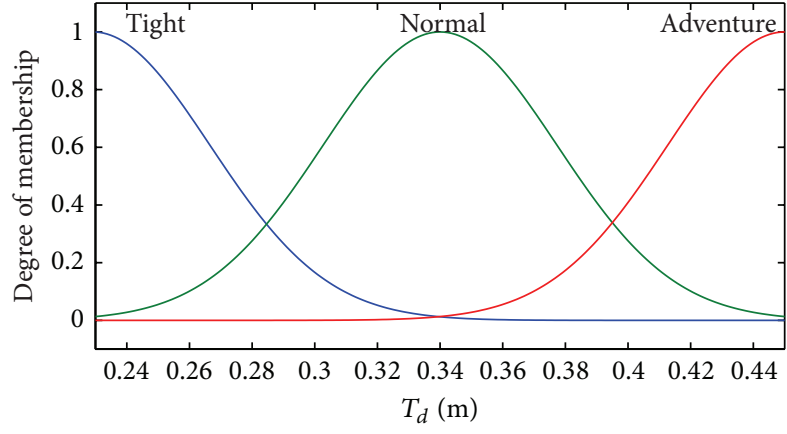

(a)

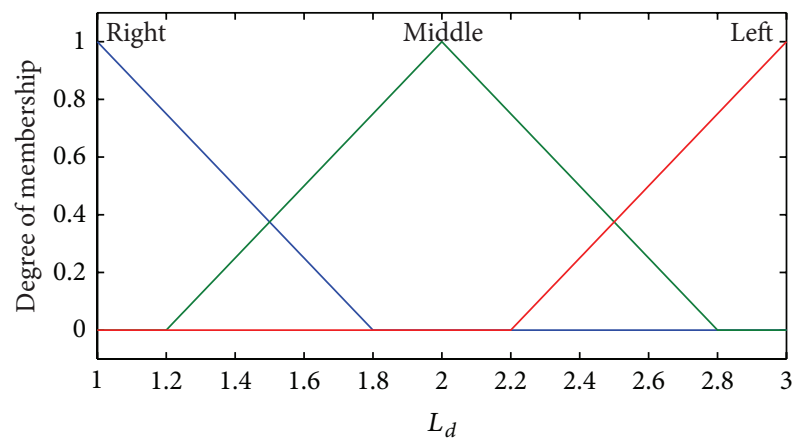

(b)

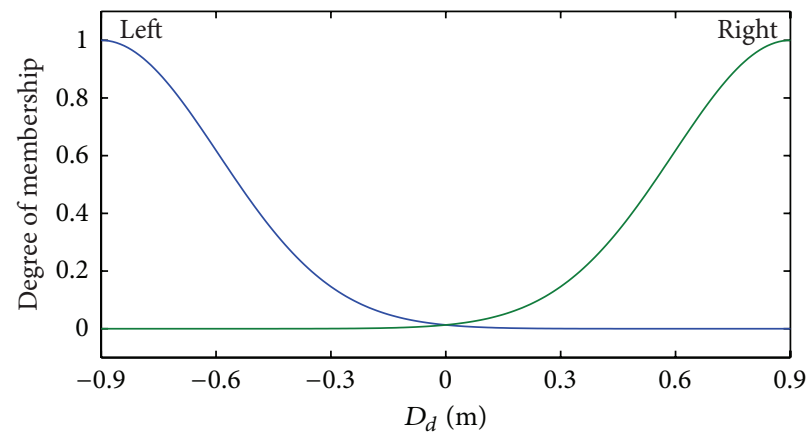

(c)

FIGURE 7: (a) Input membership function for $T_{d}$. (b) Input membership function for $L_{d}$. (c) Input membership function for $D_{d}$.

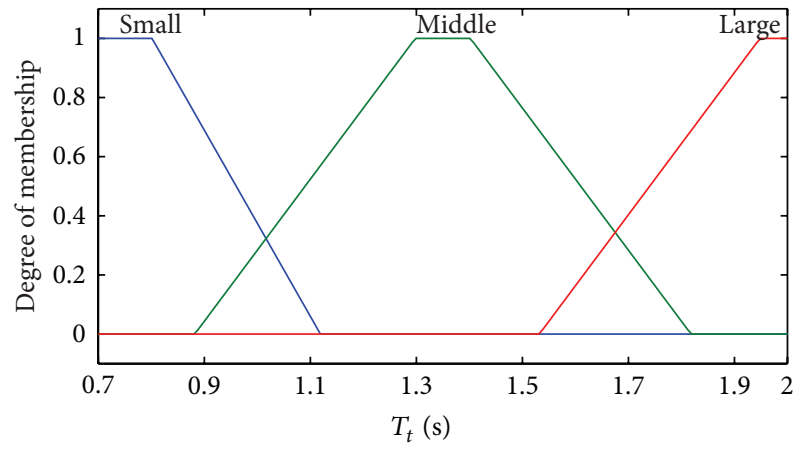

FIgURE 8: Output membership function for $T_{t}$.

and $5.4 \mathrm{~m}$ identifies a single lane change. Furthermore, the heading angle could not rise to a large value since considering lane-chang comfort. So, we determined that a value of $3^{\circ}$ is the threshold to identify a lane change. If an event has a small change in heading angle less than $3^{\circ}$ that was not lane change it should be either a lane correction or wandering on a straight road.

4.2. Turn or Curve Steering. It is slightly difficult to distinguish between turn and curve. Turn and curve are distinct in two ways: a curve's radius of curvature $R$ is much greater than that of a turn; the threshold can set $42 \mathrm{~m}$ [17]; a heading angle changes in a turn greater than curve (greater than $30^{\circ}$ for a
TABLE 2: Thresholds and criteria for identifying vehicle movement.

\begin{tabular}{lcc}
\hline Movement type & Variable & Threshold \\
\hline \multirow{2}{*}{ Lane change } & Lateral position & $1.8 \mathrm{~m} \sim 5.4 \mathrm{~m}$ \\
& Heading angle & $>3^{\circ}$ \\
\hline \multirow{2}{*}{ Turn } & Heading angle & $>30^{\circ}$ \\
& Radius of curvature & $<42 \mathrm{~m}$ \\
\hline \multirow{2}{*}{ Curve } & Heading angle & $<30^{\circ}$ \\
& Radius of curvature & $>42 \mathrm{~m}$ \\
\hline
\end{tabular}

turn and less than $30^{\circ}$ for a curve). The radius of curvature can be obtained:

$$
R=\frac{v}{w}
$$

with $v$ being the speed of vehicle and $w$ being the yaw rate.

Table 2 enumerates the thresholds and criteria described in this section.

Based on the abovementioned (Section 2.3), the detailed warning strategy is presented by the flowchart in Figure 9. Computation of TLC and determination of TLC threshold are the key of the warning strategy of LDW algorithm. The former can be calculated by the proposed new algorithm. The latter can be obtained by the fuzzy inference method described in previous section based on driver style, lane style, and departure direction inputs. What is more, in order to 


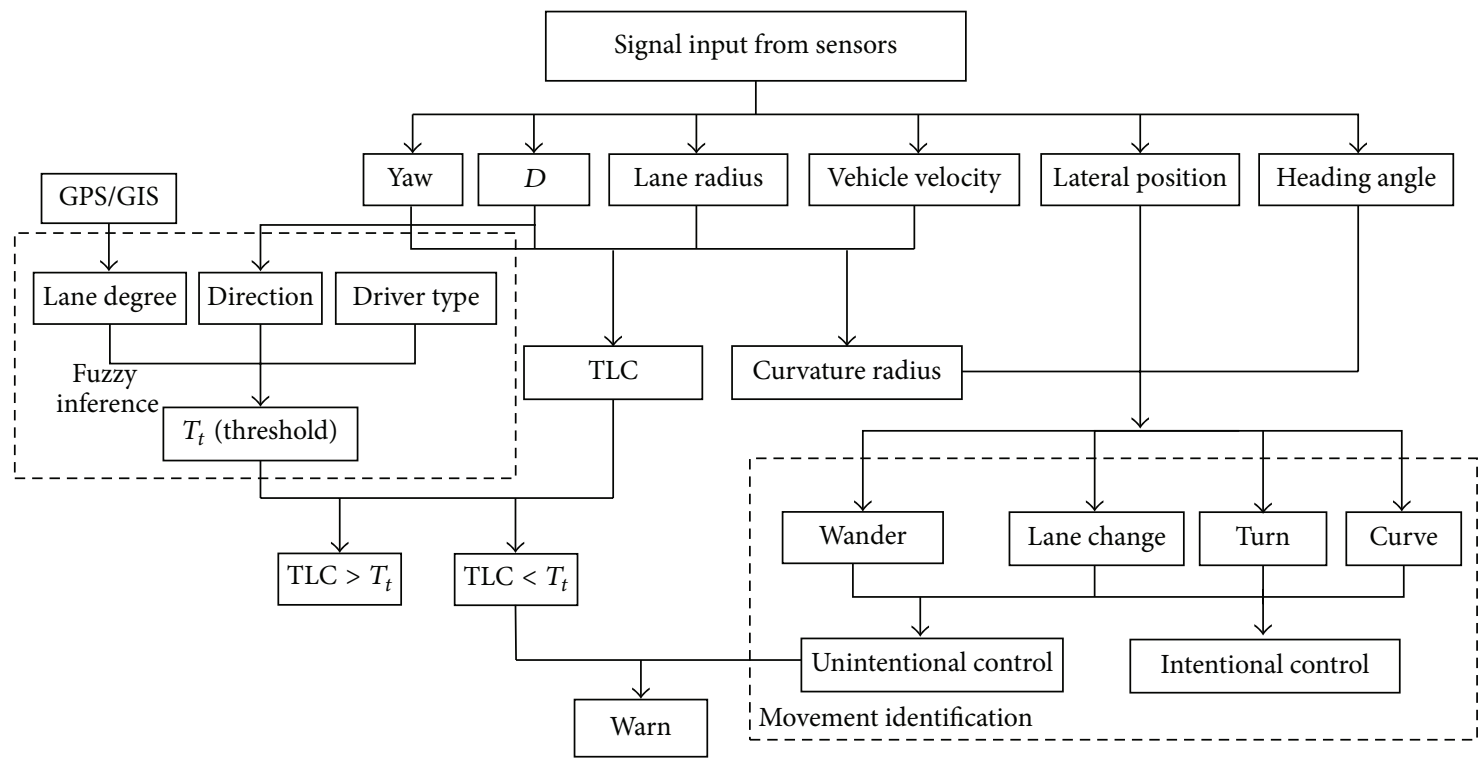

$D$ is the distance between the outside edge of the tire and lane boundary

FIGURE 9: Warning strategy of LDW algorithm.

TABLE 3: Summary of results for LDW algorithm.

\begin{tabular}{lcccccc}
\hline \multirow{2}{*}{ LDW algorithm } & \multicolumn{2}{c}{ Warning times } & \multicolumn{2}{c}{ Satisfaction (100 scores) } \\
& Unintentional & Normally & Lane change & Turn & Curve & 65 \\
The existing algorithm & 5 & 16 & 4 & 2 & 2 & 92 \\
The proposed algorithm & 5 & 9 & 0 & 0 & 0 & 27 \\
Reduce the false alarm & 0 & 7 & 4 & 2 & 2 & \\
\hline
\end{tabular}

trigger fewer nuisance alarms, a LDW strategy that determines driver's intentions by introducing identifying vehicle movements is significant. If the TLC falls below a threshold during unintentional drifting towards the lane boundary or not deliberate lane changing, a warning is triggered.

\section{Discussion of Results}

At last, the authors compared the new LDW algorithm proposed in this paper with the existing method by the real vehicle experiment. The experiment equipment and participants were the same as the abovementioned. All drivers were asked to drive on the basis of their driving habits and allowed to change lane, turn, and negotiate a curve. They were asked to drive at a favorite speed below the lane speed limit. All data were sampled with a frequency of $10 \mathrm{~Hz}$. Subjects drove continuously for $1 \mathrm{~h}$ at least. These results were summarized in Table 3.

First, we look at the ability of the LDW algorithm to detect actual lane boundary exceedance under the unintentional driving (drowsiness or distraction). The results show that the proposed algorithm is highly accurate as the existing algorithm. It is concluded that the algorithm is effective to alert drivers to departure situations for unintentional lane departure.

Next, we look at the ability of the LDW algorithm to detect actual lane boundary exceedance during normally driving. The results show that the proposed algorithm is more accurate than the existing algorithm, which minimizes nuisance alarms as far as possible compared with the latter. One of the major reasons is that the proposed LDW algorithm can detect intentional lane excursions with high success rate by identifying vehicle movements, while the existing algorithm cannot.

Finally, the satisfaction degree of the proposed algorithm (satisfaction 92) was significantly higher than the existing algorithm (satisfaction 65). This was because the proposed algorithm considered the difference of driving style, and the threshold was set comprehensively analytically (see in Section 3) rather than empirically. What is more, the higher false alarm rate can decrease driver satisfaction.

\section{Conclusions}

The results from this LDW algorithm, combined with the existing algorithm, will provide a highly reliable and practical 
method to alleviate the roadway departure problem. As stated at the beginning of the paper, the proposed LDW algorithm has the following advantages:

(i) The algorithm of TLC approximating is highly accurate compared to other algorithms mentioned. The big advantage of this algorithm over others is that it builds mathematical model of vehicle movement by integral method, makes reasonable assumption, and utilizes only relatively easy to measure variables: " $D$," " $v$," and " $\alpha . "$

(ii) The threshold of TLC is set flexibility instead of constant since considering the difference of the vehicle lateral position for different driver types or different lane, which it is likelier to be accepted by different drivers and applies to various occasions.

(iii) The algorithm can decrease false alarm rate effectively because of distinguishing from intentional lane departure, whether it is lane changes maneuvers, turn maneuvers, or negotiating a curve.

In a word, this paper seeks to draw attention to the development of LDW algorithm that can be appropriate for different drivers and situations, which approaches the goals of fewer false warnings and more warning anticipation.

\section{Conflict of Interests}

The authors declare that there is no conflict of interests regarding the publication of this paper.

\section{Acknowledgment}

The authors thank the National Natural Science Foundation of China (Grant no. 61263024).

\section{References}

[1] W. H. Wang, F. C. Sun, Q. Cao, and S. Y. Liu, Theory and Method of Driving Behavior under Road Traffic System, Science Press, Beijing, China, 2001.

[2] E. Dagan, O. Mano, G. P. Stein, and A. Shashua, "Forward collision warning with a single camera," in Proceedings of the IEEE Intelligent Vehicles Symposium, pp. 59-65, 2004.

[3] D. Pomerleau, C. Thorpe, D. Pape et al., "Run-off-road collision avoidance using IVHS countermeasures," Report DOT HS 809 170, National Highway Traffic Safety Administration, 1999.

[4] Y.Zhou, R. Xu, X.-F. Hu, and Q.-T. Ye, "A lane departure warning system based on virtual lane boundary," Journal of Information Science and Engineering, vol. 24, no. 1, pp. 293-305, 2008.

[5] T. Pilutti and A. G. Ulsoy, "Fuzzy-logic-based virtual rumble strip for road departure warning systems," IEEE Transactions on Intelligent Transportation Systems, vol. 4, no. 1, pp. 1-12, 2003.

[6] P. H. Batavia, Driver-adaptive lane departure warning systems [Dissertation], Robotics Institute, Carnegie Mellon University, Pittsburgh, Pa, USA, 1999.

[7] C. Kreucher, S. Lakshmanan, and K. Kluge, "A driver warning system based on the LOIS lane detection algorithm," in Proceedings of the IEEE International Conference on Intelligent Vehicles, pp. 17-22, Stuttgart, Germany, 1998.
[8] P. H. Batavia, D. A. Pomerleau, and C. E. Thorpe, "Predicting lane position for roadway departure prevention," in Proceedings of the IEEE Intelligent Vehicles Symposium, pp. 245-251, Stuttgart, Germany, 1998.

[9] H. Godthelp, P. Milgram, and G. J. Blaauw, "The development of a time-related measure to describe driving strategy," Human Factors, vol. 26, no. 3, pp. 257-268, 1984.

[10] W. van Winsum, K. A. Brookhuis, and D. de Waard, "A comparison of different ways to approximate time-to-line crossing (TLC) during car driving," Accident Analysis and Prevention, vol. 32, no. 1, pp. 47-56, 2000.

[11] M. Shuiwen, Modeling and Simulation foe Lane Departure Waring System, Kunming University of Science and Technology, Kunming, China, 2013.

[12] S. Lee, W. Kwon, and J.-W. Lee, "A vision based lane departure warning system," in Proceedings of the IEEE/RSJ International Conference on Intelligent Robots and Systems (IROS '99), pp. 160-165, IEEE, October 1999.

[13] Z. W. Kim, "Robust lane detection and tracking in challenging scenarios," IEEE Transactions on Intelligent Transportation Systems, vol. 9, no. 1, pp. 16-26, 2008.

[14] H. Qin, Research in the key technologies of lane departure warning system based on driving behaviors [Ph.D. thesis], Jiangsu University, Zhenjiang, China, 2014.

[15] E. Tian, Q. Xiao, X. Lu, and Z. Zhao, "Determination of alarm threshold in safe driving system with lateral warning," Journal of Chang'an University (Natural Science Edition), vol. 32, no. 1, pp. 89-94, 2012.

[16] Y. Zhou, Several key problems research of the intelligent vehicle [Ph.D. thesis], Shanghai Jiao Tong University, Shanghai, China, 2007.

[17] G. Ayres, B. Wilson, and J. LeBlanc, "Method for identifying vehicle movements for analysis of field operation test data," Journal of the Transportation Research Board, no. 1886, pp. 92-100, 2004, TRB,Nation Research Council, Washington, DC, USA. 


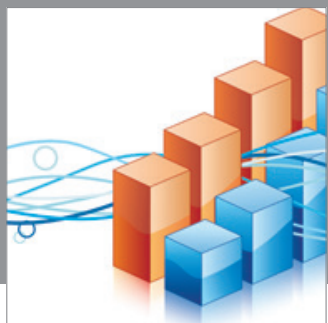

Advances in

Operations Research

mansans

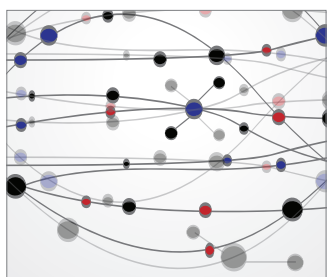

The Scientific World Journal
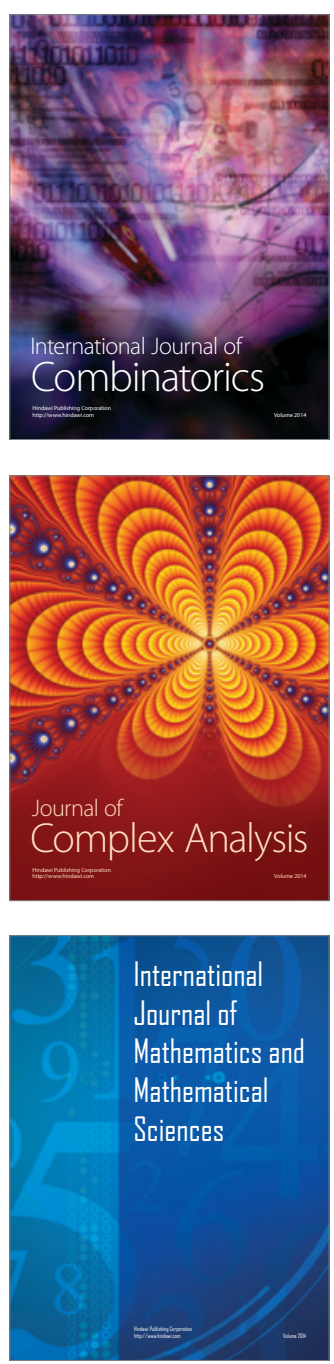
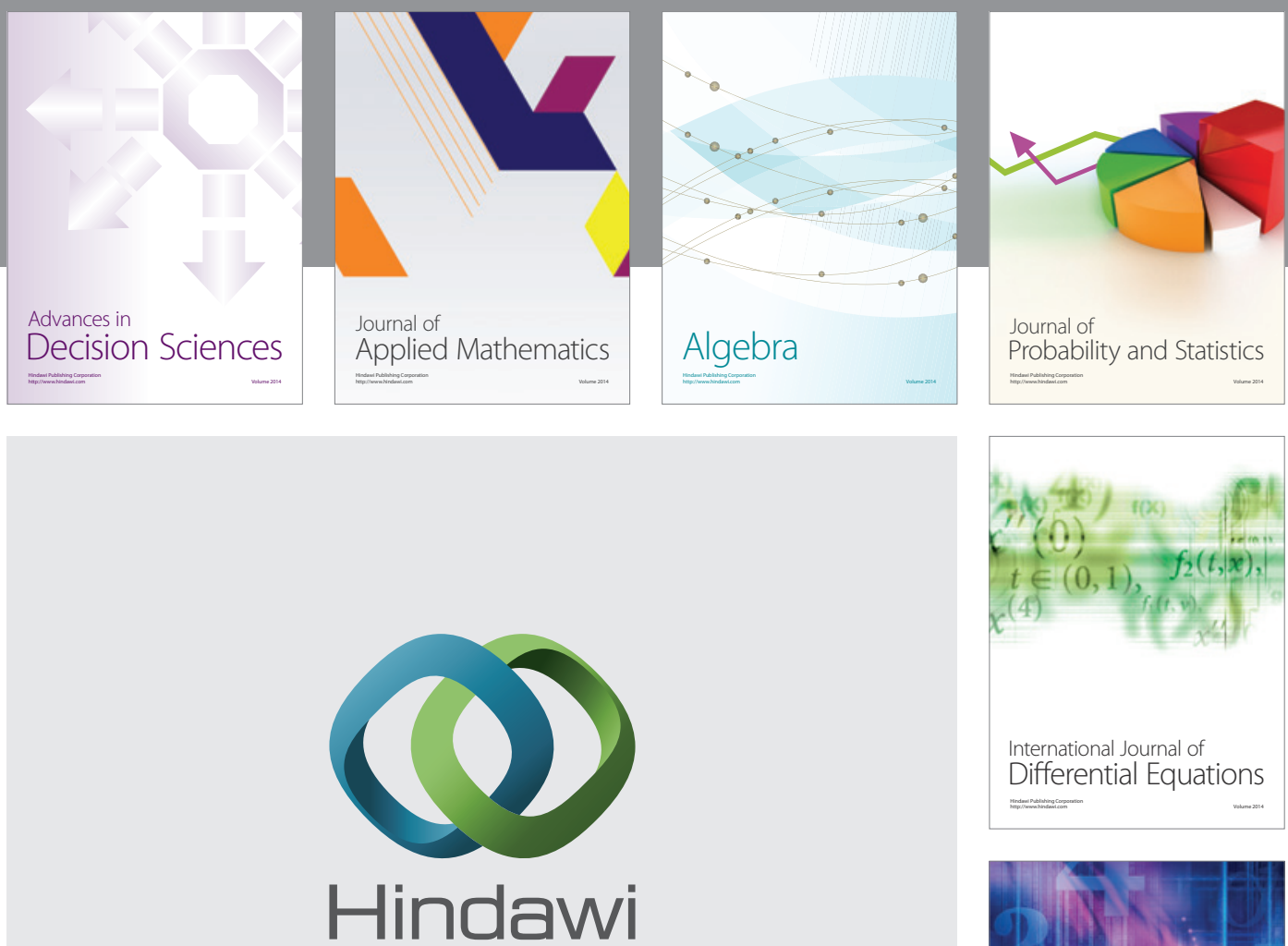

Submit your manuscripts at http://www.hindawi.com
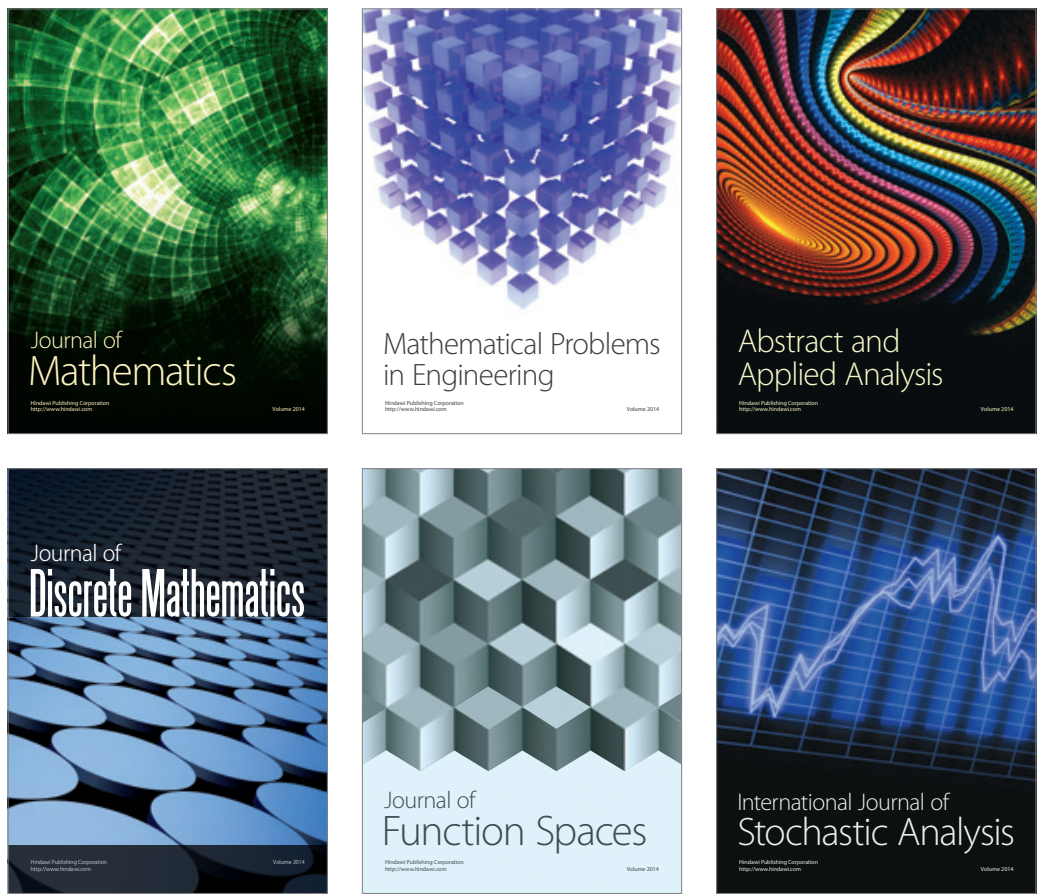

Journal of

Function Spaces

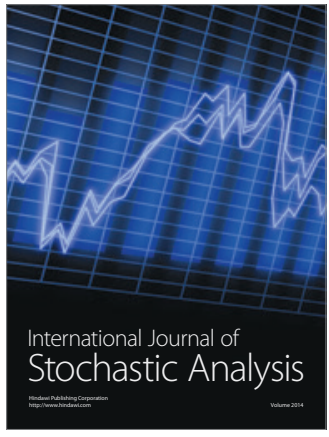

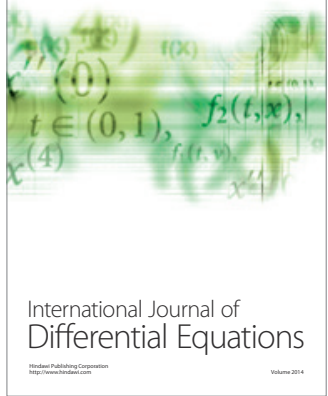
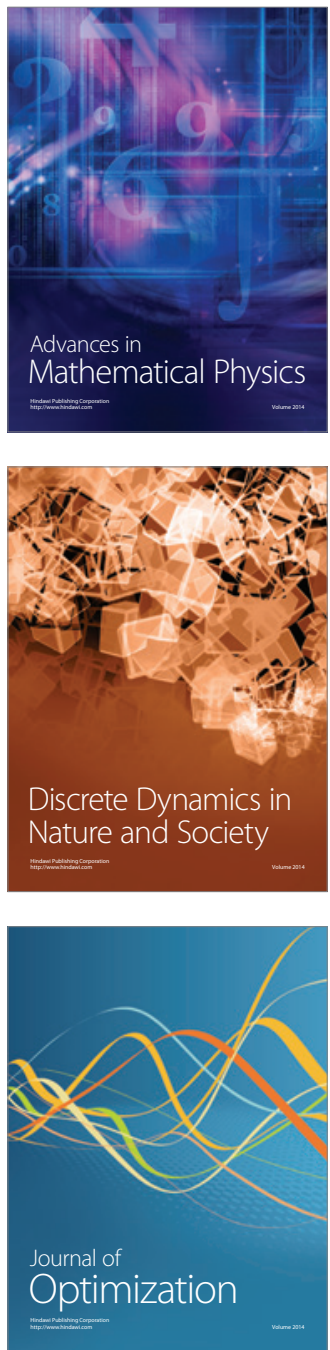